\title{
Correlations of multiplicities in length spectra for congruence subgroups
}

\author{
Yasufumi Hashimoto *
}

$\|$

\begin{abstract}
Bogomolny-Leyvraz-Schmit (1996) and Peter (2002) proposed an asymptotic formula for the correlation of the multiplicities in length spectrum on the modular surface, and Lukianov (2007) extended its asymptotic formula to the Riemann surfaces derived from the congruence subgroup $\Gamma_{0}(n)$ and the quaternion type co-compact arithmetic groups. The coefficients of the leading terms in these asymptotic formulas are described in terms of Euler products over prime numbers, and they appear in eigenvalue statistic formulas found by Rudnick (2005) and Lukianov (2007) for the Laplace-Beltrami operators on the corresponding Riemann surfaces. In the present paper, we further extend their asymptotic formulas to the higher level correlations of the multiplicities for any congruence subgroup of the modular group.
\end{abstract}

\section{Introduction}

It is known that there are deep connections between the geometry on hyperbolic manifolds and the spectra of the Laplace-Beltrami operators on the corresponding manifolds. In fact, two compact Riemann surface have the same length spectra if and only if the Laplacians on these surfaces have the same spectra [5, 15]. And the Selberg trace formula describes a relation between the lengths of the primitive closed geodesics and the eigenvalues of the Laplace-Beltrami operator [31]. Such a situation is quite similar to the quantum theory; Gutzwiller's trace formula describes connections between the periodic orbits in the classical models and the energy spectra in the quantum models, although its rigorous proof has not been given yet [11].

The asymptotic formula, called the prime geodesic theorem, of the number of the primitive closed geodesics on a hyperbolic manifold with the finite volume is given due to Selberg's trace formula [31, 7, 8]. This is interpreted as an analogue of the prime number theorem which is the asymptotic formula for the number of rational prime numbers, since the leading terms of both formulas are similar and the error terms are given by the non-trivial zeros

*Department of Mathematical Science, University of the Ryukyus, Nishihara-cho, Okinawa 903-0213, Japan. e-mail: hashimoto@math.u-ryukyu.ac.jp

MSC2010: primary: 11M36; secondary: 11F72

Keywords: length spectrum, congruence subgroups, prime geodesic theorem, class numbers 
of the corresponding zeta functions. However, the detail distributions of the prime numbers and the primitive geodesics are different. In fact, there are many geodesics with the same lengths in Riemann surfaces and the number of geodesics with the same length (the multiplicity in the length spectrum) is unbounded [24], despite there are no such primes. The results on numerical experiments imply that (the fundamental group of) the manifold is arithmetic if and only if the multiplicity is highly increasing, and some experts on arithmetic quantum chaos pointed out that such a phenomenon is strongly connected to the observations that the eigenvalue statistics of the Laplacian seems to be the Poisson distribution on arithmetic surfaces, and the Gaussian orthogonal ensemble (GOE) on non-arithmetic surfaces [2, 3, 18, 21]. Note that any theoretical proof for such situations has not been given yet (see [33, 19, 29, 10]).

The aim in the present paper is to study the growth of the multiplicity in length spectra for Riemann surfaces derived from congruence subgroups of the modular group. Though writing down the length spectra and its multiplicity with elementary objects is not easy in general, the multiplicities for the congruence subgroups are written in terms of the class numbers of indefinite binary quadratic forms [27, 6]. Applying results and approaches for the class numbers in the classical analytic number theory, Bogomolny-Leyvraz-Schmit [3] and Peter [23] presented the asymptotic formulas for the sum of the shifted product of multiplicities for the modular surface, whose coefficient in the leading term is explicitly drawn as an Euler product over prime numbers. Lukianov [17] has obtained similar asymptotic formulas for the Riemann surface whose fundamental group is $\Gamma_{0}(n)$, a congruence subgroup of the modular group, or a co-compact arithmetic group derived from the indefinite quaternion algebra. Rudnick [26] and Lukianov [17] found that the coefficient of the leading term in the asymptotic formulas for the square sum of the multiplicities in length spectra appear in the formula for statistic behavior of the eigenvalues of the Laplace-Beltrami operators.

The main result in this paper is to propose asymptotic formulas for the sum of higher shifted product of the multiplicities in length spectrum for any congruence subgroup of the modular group with an explicit description of the coefficient of their leading term, which is an extension of the results in [3, 23, 17]. Since the multiplicities are given by the class numbers of quadratic forms, they are approximated by periodic functions [23]. Thus, using the approaches in the theory of arithmetic functions [30], we can get the leading terms of the asymptotic formulas for the products of multiplicities and the expressions of the coefficients of the leading terms as products over prime numbers.

The basic notations and the main result in the present paper are as follows.

Notations and the main result. Let $H:=\{x+y \sqrt{-1}, y>0\}$ be the upper half plane and $\Gamma$ a discrete subgroup of $\mathrm{SL}_{2}(\mathbb{R})$ with $\operatorname{vol}(\Gamma \backslash H)<\infty$. Denote by $\operatorname{Prim}(\Gamma)$ the set of primitive hyperbolic conjugacy classes of $\Gamma$ and $N(\gamma)$ the square of the larger eigenvalue of $\gamma \in \operatorname{Prim}(\Gamma)$. It is known that

$$
\pi_{\Gamma}(x):=\#\{\gamma \in \operatorname{Prim}(\Gamma) \mid N(\gamma)<x\} \sim \operatorname{li}(x) \quad \text { as } \quad x \rightarrow \infty,
$$

where $\operatorname{li}(x):=\int_{2}^{x}(\log t)^{-1} d t$. Since there is a one-to-one correspondence between elements in Prim $(\Gamma)$ and primitive closed geodesics on $\Gamma \backslash H$ and $N(\gamma)$ coincides with the exponential of the length of the corresponding geodesic, the asymptotic formula (1.1) is called the prime geodesic theorem (see, e.g. [31, 14]).

Let $\operatorname{Tr}(\Gamma)$ be the set of $\operatorname{tr} \gamma$ of $\gamma \in \operatorname{Prim}(\Gamma)$ and $m_{\Gamma}(t)$ for $t \in \operatorname{Tr} \Gamma$ be the number of $\gamma \in$ 
$\operatorname{Prim}(\Gamma)$ with $\operatorname{tr} \gamma=t$. Since $\operatorname{tr} \gamma=N(\gamma)^{1 / 2}+N(\gamma)^{-1 / 2}$ and $N(\gamma)^{1 / 2}=\left(\operatorname{tr} \gamma+\sqrt{(\operatorname{tr} \gamma)^{2}-4}\right) / 2$, the set $\left\{\left(t, m_{\Gamma}(t)\right)\right\}_{t \in \operatorname{Tr}(\Gamma)}$ is identified to the length spectrum on $\Gamma \backslash H$ and the prime geodesic theorem (1.1) is written by

$$
\pi_{\Gamma}\left(x^{2}\right)=\sum_{\substack{t \in \operatorname{Tr}(\Gamma) \\ t<x}} m_{\Gamma}(t) \sim \operatorname{li}\left(x^{2}\right) .
$$

It is obvious that $\operatorname{Tr}\left(\mathrm{SL}_{2}(\mathbb{Z})\right)=\mathbb{Z}_{\geq 3}$ and $\operatorname{Tr}(\Gamma) \subset \mathbb{Z}_{\geq 3}$ for $\Gamma \subset \mathrm{SL}_{2}(\mathbb{Z})$. Then the prime geodesic theorem (1.2) for such $\Gamma$ is given as a sum over integers.

$$
\pi_{\Gamma}\left(x^{2}\right)=\sum_{3 \leq t<x} m_{\Gamma}(t) \sim \operatorname{li}\left(x^{2}\right) .
$$

For $\Gamma=\mathrm{SL}_{2}(\mathbb{Z})$ and an integer $r \geq 0$, Bogomolny-Leyvraz-Schmit [3] and Peter [23] presented the following asymptotic formula.

$$
\pi_{\mathrm{SL}_{2}(\mathbb{Z})}^{(2)}\left(x^{2} ; r\right):=\sum_{3 \leq t<x} m_{\mathrm{SL}_{2}(\mathbb{Z})}(t) m_{\mathrm{SL}_{2}(\mathbb{Z})}(t+r) \sim c_{\mathrm{SL}_{2}(\mathbb{Z})}^{(2)}(r) \mathrm{li}_{2}\left(x^{3}\right),
$$

where $\operatorname{li}_{2}(x):=\int_{2}^{x}(\log t)^{-2} d t$ and $c_{\mathrm{SL}_{2}(\mathbb{Z})}^{(2)}(r)$ is a constant described in [3, 23] as a product over prime numbers. Lukianov [17 also proposed asymptotic formulas for $r=0$ and for $\Gamma=\Gamma_{0}(n)$ with square free $n$ or a quaternion type co-compact arithmetic $\Gamma$.

The aim in the present paper is to extend the asymptotic formula (1.4) to the higher shifted product of the multiplicities for any congruence subgroup. For an integer $n \geq 1$, let $\mathbb{Z}_{n}:=\mathbb{Z} / n \mathbb{Z}$ and

$$
\begin{aligned}
& \Gamma(n):=\operatorname{Ker}\left(\mathrm{SL}_{2}(\mathbb{Z}) \stackrel{\text { proj. }}{\rightarrow} \mathrm{SL}_{2}\left(\mathbb{Z}_{n}\right)\right)=\left\{\gamma \in \mathrm{SL}_{2}(\mathbb{Z}) \mid \gamma \equiv I \bmod n\right\}, \\
& \hat{\Gamma}(n):=\operatorname{Ker}\left(\mathrm{SL}_{2}(\mathbb{Z}) \stackrel{\text { proj. }}{\rightarrow} \mathrm{PSL}_{2}\left(\mathbb{Z}_{n}\right)\right)=\left\{\gamma \in \mathrm{SL}_{2}(\mathbb{Z}) \mid \gamma \equiv \alpha I \bmod n, \alpha^{2} \equiv 1 \bmod n\right\},
\end{aligned}
$$

the principal congruence subgroups of level $n$. Throughout in this paper, we call $\Gamma$ a congruence subgroup of level $n$ if $\hat{\Gamma}(n) \subset \Gamma \subset \mathrm{SL}_{2}(\mathbb{Z})$ and $\Gamma \not \supset \hat{\Gamma}(m)$ for any $m<n$.

The main result in this paper is to extend (1.4) as follows.

Theorem 1.1. Let $k \geq 2$ be an integer, $\mathbf{r}:=\left(r_{1}, \cdots, r_{k}\right) \in \mathbb{Z}^{k}$ and $\Gamma$ a congruence subgroup of $\mathrm{SL}_{2}(\mathbb{Z})$. Then we have

$$
\pi_{\Gamma}^{(k)}\left(x^{2} ; \mathbf{r}\right):=\sum_{3 \leq t<x} m_{\Gamma}\left(t+r_{1}\right) \cdots m_{\Gamma}\left(t+r_{k}\right) \sim c_{\Gamma}^{(k)}(\mathbf{r}) \operatorname{li}_{k}\left(x^{k+1}\right),
$$

where $\mathrm{li}_{k}(x):=\int_{2}^{x}(\log t)^{-k} d t$ and $c_{\Gamma}^{(k)}(\mathbf{r})$ is a constant described in Theorem 3.1.

\section{Length spectra for congruence subgroups}

In this section, we propose an expression of the multiplicities in length spectrum for a congruence subgroup in terms of indefinite binary quadratic forms.

\subsection{Quadratic forms and the modular group}

Let $Q(x, y)=[a, b, c]:=a x^{2}+b x y+c y^{2}$ be a binary quadratic form over $\mathbb{Z}$ with $a, b, c \in \mathbb{Z}$ and $\operatorname{gcd}(a, b, c)=1$. Denote by $D=D(Q):=b^{2}-4 a c$ the discriminant of $[a, b, c]$. We call quadratic forms $Q$ and $Q^{\prime}$ equivalent and write $Q \sim Q^{\prime}$ if there exists $g \in \mathrm{SL}_{2}(\mathbb{Z})$ such that 
$Q(x, y)=Q^{\prime}((x, y) \cdot g)$. Denote by $h(D)$ the number of equivalence classes of the quadratic forms of given $D=b^{2}-4 a c$. It is known that, if $D>0$, then there are infinitely many positive solutions $(t, u)$ of the Pell equation $t^{2}-D u^{2}=4$. Put $\left(t_{j}, u_{j}\right)=\left(t_{j}(D), u_{j}(D)\right)$ the $j$-th positive solution of $t^{2}-D u^{2}=4$ and $\epsilon_{j}(D):=\left(t_{j}(D)+u_{j}(D) \sqrt{D}\right) / 2$. Note that $\epsilon(D)=\epsilon_{1}(D)$ is called the fundamental unit of $D$ in the narrow sense, and it holds that $\epsilon_{j}(D)=\epsilon(D)^{j}$.

For a quadratic form $Q=[a, b, c]$ and a positive solution $(t, u)$ of $t^{2}-D u^{2}=4$, let

$$
\gamma(Q,(t, u)):=\left(\begin{array}{cc}
\frac{t+b u}{2} & -c u \\
a u & \frac{t-b u}{2}
\end{array}\right) \in \mathrm{SL}_{2}(\mathbb{Z}) .
$$

Conversely, for $\gamma=\left(\gamma_{i j}\right)_{1 \leq i, j \leq 2} \in \mathrm{SL}_{2}(\mathbb{Z})$, we put

$$
\begin{aligned}
& t_{\gamma}:=\gamma_{11}+\gamma_{22}, \quad u_{\gamma}:=\operatorname{gcd}\left(\gamma_{21}, \gamma_{11}-\gamma_{22},-\gamma_{12}\right), \\
& a_{\gamma}:=\gamma_{21} / u_{\gamma}, \quad b_{\gamma}:=\left(\gamma_{11}-\gamma_{22}\right) / u_{\gamma}, \quad c_{\gamma}:=-\gamma_{12} / u_{\gamma}, \\
& Q_{\gamma}:=\left[a_{\gamma}, b_{\gamma}, c_{\gamma}\right], \quad D_{\gamma}:=\frac{t_{\gamma}^{2}-4}{u_{\gamma}^{2}}=b_{\gamma}^{2}-4 a_{\gamma} c_{\gamma} .
\end{aligned}
$$

It is known that (2.1) and (2.2) give a one-to-one correspondence between equivalence classes of primitive binary quadratic forms with $D>0$ and primitive hyperbolic conjugacy classes of $\mathrm{SL}_{2}(\mathbb{Z})$ (see [27] and Chap. 5 in [9]). Then the multiplicity $m_{\Gamma}(t)$ for $\Gamma=\mathrm{SL}_{2}(\mathbb{Z})$ is described as follows (see, e.g. [27]).

Proposition 2.1. Let $t \geq 3$ be an integer. Then we have

$$
m_{\mathrm{SL}_{2}(\mathbb{Z})}(t)=\sum_{u \in U(t), j_{t, u}=1} h\left(D_{t, u}\right),
$$

where $D_{t, u}:=\left(t^{2}-4\right) / u^{2}, U(t):=\left\{u \geq 1\left|u^{2}\right| t^{2}-4, D_{t, u} \in \mathfrak{D}\right\}$ and

$$
j_{t, u}:=\max \left\{j \geq 1 \mid \epsilon_{j}\left(D_{t, u}\right)=\frac{1}{2}\left(t+\sqrt{t^{2}-4}\right)\right\} \text {. }
$$

\subsection{Conjugations in $\mathrm{PSL}_{2}(\mathbb{Z} / n \mathbb{Z})$}

For an integer $n \geq 1$, let $\mathbb{Z}_{n}^{*}$ be the multiplicative group in $\mathbb{Z}_{n}:=\mathbb{Z} / n \mathbb{Z}$ and $\mathbb{Z}_{n}^{(2)}:=\mathbb{Z}_{n}^{*} /\left(\mathbb{Z}_{n}^{*}\right)^{2}$. Note that

$$
\mathbb{Z}_{p^{r}}^{(2)}= \begin{cases}\{1,3,5,7\}, & (p=2, r \geq 3), \\ \{1, \eta\}, & (p \geq 3, r \geq 1),\end{cases}
$$

where $\eta$ is a non quadratic residue of $p$.

Lemma 2.2. Let $n \geq 1, a, b, c$ be integers with $\operatorname{gcd}(a, b, c, n)=1$. Denote by $D:=b^{2}-4 a c$ and suppose that $t, u \geq 1$ satisfies $t^{2}-D u^{2}=4$. Put

$$
\gamma:=\left(\begin{array}{cc}
\frac{1}{2}(t+b u) & -c u \\
a u & \frac{1}{2}(t-b u)
\end{array}\right), \quad \gamma_{\nu}=\gamma_{\nu}(D):=\left(\begin{array}{cc}
\frac{t+\delta u}{2} & \frac{D-\delta^{2}}{4} \nu^{-1} u \\
\nu u & \frac{t-\delta u}{2}
\end{array}\right)
$$

where $\nu \in \mathbb{Z}_{n}^{*}$, and $\delta$ is 1 when $D \equiv 1 \bmod 4$ and is 0 otherwise. Then, for any $\gamma \in \mathrm{SL}_{2}(\mathbb{Z})$, there exists $\nu \in \mathbb{Z}_{n}^{(2)}$ such that $\gamma \sim \gamma_{\nu}$ in $\operatorname{PSL}_{2}\left(\mathbb{Z}_{n}\right)$. 
multiplicities in length spectra for congruence subgroups

Proof. When $\operatorname{gcd}(a, n) \neq 1$, it is easy to see that there exists $g^{\prime} \in \mathrm{PSL}_{2}\left(\mathbb{Z}_{n}\right)$ such that $\operatorname{gcd}\left(\left(g^{\prime-1} \gamma g^{\prime}\right)_{21} / u, n\right)=1$. Then the problem is reduced to the case of $\operatorname{gcd}(a, n)=1$. When $\operatorname{gcd}(a, n)=1$, put $\alpha \in \mathbb{Z}_{n}^{*}$ and $\nu \in \mathbb{Z}_{n}^{(2)}$ such that $a=\nu \alpha^{2}$. We have $g^{-1} \gamma g=\gamma_{\nu}$ where

$$
g:=\left(\begin{array}{cc}
\alpha^{-1} & (\nu \alpha)^{-1}(b+\delta) / 2 \\
0 & \alpha
\end{array}\right) .
$$

Thus the claim in Lemma 2.2 holds.

Lemma 2.3. Let $p$ be a prime and $r \geq 1$ an integer. Then, for any $\nu \in \mathbb{Z}_{p^{r}}^{(2)}$, there exists $l_{1}, l_{2} \geq 1$ such that $\gamma_{1}^{l_{1}} \sim \gamma_{\nu}$ and $\gamma_{1} \sim \gamma_{\nu}^{l_{2}}$ in $\mathrm{PSL}_{2}\left(\mathbb{Z}_{p^{r}}\right)$.

Proof. The case of $p=2$. If $2 \nmid t$ then $D \equiv 5 \bmod 8$ and $\delta=1$ since $D u^{2}=t^{2}-4$. In this case, we see that

$$
\left(g_{2}^{-1} \gamma_{1} g_{2}\right)_{21} / u \equiv 7, \quad\left(g_{4}^{-1} \gamma_{1} g_{4}\right)_{21} / u \equiv 3, \quad\left(g_{6}^{-1} \gamma_{1} g_{6}\right)_{21} / u \equiv 5 \bmod 8,
$$

where $g_{i}:=\left(\begin{array}{ll}1 & 0 \\ i & 1\end{array}\right)$. Thus, according to Lemma 2.2, we have

$$
2 \nmid t \Rightarrow \gamma_{1} \sim \gamma_{3} \sim \gamma_{5} \sim \gamma_{7} \quad \text { in } \operatorname{PSL}_{2}\left(\mathbb{Z}_{2^{r}}\right) .
$$

If $4 \mid t$ then $D \equiv-4 \bmod 16$ and $\delta=0$. In this case, we see that

$$
\left(g_{2}^{-1} \gamma_{1} g_{2}\right)_{21} / u \equiv 5, \quad\left(g_{2}^{-1} \gamma_{3} g_{2}\right)_{21} / u \equiv 7 \bmod 8,
$$

namely

$$
4 \mid t \quad \Rightarrow \quad \gamma_{1} \sim \gamma_{5}, \quad \gamma_{3} \sim \gamma_{7}
$$

Since $\left(t_{j}+u_{j} \sqrt{D}\right) / 2=((t+u \sqrt{D}) / 2)^{j}$, we have $u_{3} / u=t^{2}-1$. This gives that $u_{3} / u_{1} \equiv$ $-1 \bmod 8$ for $4 \mid t$, and then we get

$$
4 \mid t \Rightarrow \gamma_{1}^{3} \sim \gamma_{7}, \quad \gamma_{3}^{3} \sim \gamma_{5}, \quad \gamma_{5}^{3} \sim \gamma_{3}, \quad \gamma_{7}^{3} \sim \gamma_{1} .
$$

We can similarly obtain

$$
t \equiv 2 \bmod 4 \Rightarrow \gamma_{1}^{\nu} \sim \gamma_{\nu}, \quad \gamma_{\nu}^{\nu} \sim \gamma_{1} \quad \text { for any } \nu \in \mathbb{Z}_{p^{r}}^{(2)} .
$$

The case of $p=3$. We have $\left(g^{-1} \gamma_{1} g\right)_{21} \equiv 2 u \bmod 3^{r}$, where

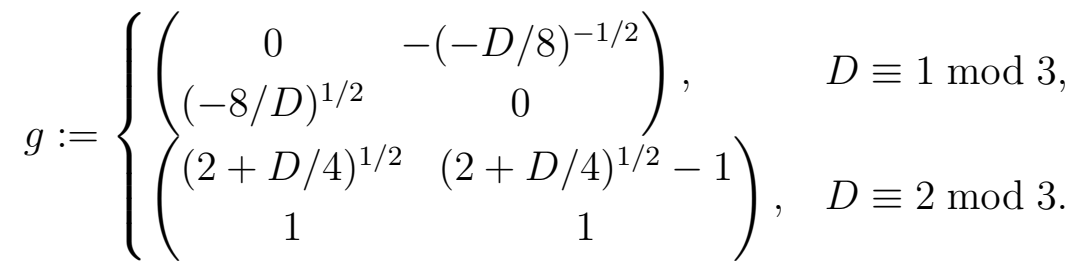

Then $\gamma_{1} \sim \gamma_{2}$ in $\mathrm{PSL}_{2}\left(\mathbb{Z}_{3^{r}}\right)$ for $3 \nmid D$. For $3 \mid D$, it is easy to see that $\gamma_{1}^{2} \sim \gamma_{2}$ and $\gamma_{2}^{2} \sim \gamma_{1}$. The case of $p \geq 5$. First, study the existence of solutions $(x, y)$ of the equation $x^{2}-$ $d y^{2} \equiv \eta \bmod p^{r}$ where $(\eta / p)=-1$. When $p \mid d$, there are no solutions. When $(d / p)=1$, $x=(\eta+1) / 2$ and $y=(\eta-1) /\left(2 d^{1 / 2}\right)^{-1}$ is a solution. When $(d / p)=-1$, let $d_{1} \equiv\left(d \eta^{-1}\right)^{1 / 2}$. Since the equation is written by $x^{2} \equiv \eta\left(1+d_{1}^{2} y^{2}\right)$, choosing $y$ such that $1+d_{1}^{2} y^{2}$ is a non quadratic residue, we see that the equation has a solution.

Thus, if $p \nmid d$, we see that $\left(g^{-1} \gamma_{1} g\right)_{21} \equiv \eta$ and then $\gamma_{1} \sim \gamma_{\eta}$, where $g$ is given such that $(x, y)=\left(g_{11}, g_{21}\right)$ is a solution of $x^{2}-d y^{2} \equiv \eta \bmod p^{r}$. If $p \mid D$, similar to the case of $p=2$, we have

$$
\frac{u_{\eta}}{u_{1}}=\sum_{l=0}^{(\eta-1) / 2}\left(\begin{array}{c}
\eta \\
2 l+1
\end{array}\right)\left(\frac{t}{2}\right)^{\eta-2 l-1}\left(t^{2}-4\right)^{l},
$$


where $\eta$ is chosen to be odd. Since $p|D| t^{2}-4$, we get

$$
\frac{u_{\eta}}{u_{1}} \equiv \eta\left((t / 2)^{(\eta-1) / 2}\right)^{2} \equiv \eta \alpha^{2}
$$

for some $\alpha \in \mathbb{Z}_{p^{r}}^{*}$. Thus it holds that $\gamma_{1}^{\eta} \sim \gamma_{\eta}$ and $\gamma_{\eta}^{\nu} \sim \gamma_{1}$ where $\eta \nu \equiv 1 \bmod p$.

\subsection{Length spectra for congruence subgroups}

According to Venkov-Zograf's formula [34] for Selberg's zeta function, we have

$$
\hat{m}_{\Gamma}(t):=\sum_{\substack{\gamma \in \operatorname{Prim}(\Gamma), j \geq 1 \\ t_{\gamma^{j}}=t}} \frac{1}{j}=\sum_{\substack{\gamma \in \operatorname{Prim}\left(\mathrm{SL}_{2}(\mathbb{Z})\right), j \geq 1 \\ t_{\gamma^{j}}=t}} \frac{1}{j} \operatorname{tr} \chi_{\Gamma}\left(\gamma^{j}\right),
$$

where $\chi_{\Gamma}:=\operatorname{Ind}_{\Gamma}^{\mathrm{SL}_{2}(\mathbb{Z})} 1$. The value $\hat{m}_{\Gamma}(t)$ is expressed as follows.

Proposition 2.4. Let $n \geq 1, t \geq 3$ be integers and $\Gamma$ a congruence subgroup of level $n$. Then we have

$$
\hat{m}_{\Gamma}(t)=\sum_{u \in U(t)} \frac{1}{j_{t, u}} \omega_{\Gamma}(t, u) h\left(D_{t, u}\right),
$$

where $\omega_{\Gamma}(t, u):=\operatorname{tr} \chi_{\Gamma}\left(\gamma_{1}\left(D_{t, u}\right)\right)$ and $\gamma_{1}\left(D_{t, u}\right)$ is given in Lemma 2.2.

Proof. First study the case of $n=p^{r}$. Due to Lemma 2.2] and 2.3, we see that, for any $\gamma, \gamma^{\prime} \in \mathrm{SL}_{2}(\mathbb{Z})$ with $D_{\gamma}=D_{\gamma^{\prime}}$, there exists $\eta, \nu \in \mathbb{Z}$ such that $\gamma^{\nu} \sim \gamma^{\prime}$ and $\gamma^{\prime \eta} \sim \gamma$ in $\operatorname{PSL}_{2}\left(\mathbb{Z}_{p^{r}}\right)$. Since $\chi_{\Gamma}=\operatorname{Ind}_{\Gamma}^{\mathrm{SL}_{2}(\mathbb{Z})} 1 \simeq \operatorname{Ind}_{\Gamma / \hat{\Gamma}\left(p^{r}\right)}^{\mathrm{PSL}_{2}\left(\mathbb{Z}_{\left.p^{r}\right)}\right.} 1$ is a permutation representation of $\operatorname{PSL}_{2}\left(\mathbb{Z}_{p^{r}}\right)$, we see that $\chi_{\Gamma}(\gamma) \sim \chi_{\Gamma}\left(\gamma^{\prime}\right)$, namely $\operatorname{tr} \chi_{\Gamma}(\gamma)$ depends only on $D_{\gamma}$. Thus (2.8) for $n=p^{r}$ follows from (2.7).

When $n$ is factored by $n=\prod_{p \mid n} p^{r}$, it holds that $\chi_{\Gamma}(\gamma)=\bigotimes_{p \mid n} \chi_{\Gamma \hat{\Gamma}\left(p^{r}\right)}(\gamma)$ (see [13]). Thus (2.8) holds also for any $n$.

\section{Proof of Theorem 1.1}

\subsection{Description of $c_{\Gamma}^{(k)}(\mathbf{r})$}

The coefficient $c_{\Gamma}^{(k)}(\mathbf{r})$ in Theorem 1.1 is described in the following theorem.

Theorem 3.1. Let $t \geq 3$ be an integer, $\Gamma$ a congruence subgroup of $\mathrm{SL}_{2}(\mathbb{Z})$ and

$$
I_{\Gamma}(t):=\frac{\log \left(\frac{1}{2}\left(t+\sqrt{t^{2}-4}\right)\right)}{\sqrt{t^{2}-4}} \hat{m}_{\Gamma}(t) .
$$

Then, for any $k \geq 1$ and $\mathbf{r}:=\left(r_{1}, \cdots, r_{k}\right) \in \mathbb{Z}^{k-1}$, the limit

exists and coincides with

$$
c_{\Gamma}^{(k)}(\mathbf{r}):=\lim _{x \rightarrow \infty} \frac{1}{x} \sum_{3 \leq t \leq x} I_{\Gamma}\left(t+r_{1}\right) \cdots I_{\Gamma}\left(t+r_{k}\right)
$$

$$
c_{\Gamma}^{(k)}(\mathbf{r})=\prod_{p}\left(\lim _{l \rightarrow \infty} p^{l(k-1)} \sum_{m \in \mathbb{Z}_{p^{l}}} F_{\Gamma}\left(m+r_{1} ; p^{l}\right) \cdots F_{\Gamma}\left(m+r_{k} ; p^{l}\right)\right)
$$


where

$$
F_{\Gamma}(m ; n):=\frac{\#\{\gamma \in \Gamma(n) \backslash \Gamma(n) \Gamma \mid \operatorname{tr} \gamma \equiv m \bmod n\}}{\# \Gamma(n) \backslash \Gamma(n) \Gamma}
$$

We now prove that Theorem 1.1 follows from Theorem 3.1 with the same $c_{\Gamma}^{(k)}(\mathbf{r})$. Proof of "Theorem $3.1 \Rightarrow$ Theorem 1.1]". Let

$$
\hat{\pi}_{\Gamma}^{(k)}(x ; \mathbf{r}):=\sum_{3 \leq t \leq x} \hat{m}_{\Gamma}\left(t+r_{1}\right) \cdots \hat{m}_{\Gamma}\left(t+r_{k}\right) .
$$

It is easy to see that if Theorem 3.1 holds then

$$
\hat{\pi}_{\Gamma}^{(k)}(x ; \mathbf{r}) \sim c_{\Gamma}^{(k)}(\mathbf{r}) \operatorname{li}_{k}\left(x^{k+1}\right) .
$$

We now compare $\pi_{\Gamma}^{(k)}(x ; \mathbf{r})$ and $\hat{\pi}_{\Gamma}^{(k)}(x ; \mathbf{r})$ as follows.

$$
\begin{aligned}
\left|\hat{\pi}_{\Gamma}^{(k)}(x ; \mathbf{r})-\pi_{\Gamma}^{(k)}(x ; \mathbf{r})\right| \leq & \sum_{1 \leq i \leq k} \sum_{3 \leq t \leq x} \hat{m}_{\Gamma}\left(t+r_{1}\right) \cdots \hat{m}_{\Gamma}\left(t+r_{i-1}\right) \\
& \times\left|\hat{m}_{\Gamma}\left(t+r_{i}\right)-m_{\Gamma}\left(t+r_{i}\right)\right| m_{\Gamma}\left(t+r_{i+1}\right) \cdots m_{\Gamma}\left(t+r_{k}\right) .
\end{aligned}
$$

The classical bound of the class numbers $h\left(D_{t, u}\right) \ll D_{t, u}^{1 / 2+\epsilon} \ll t^{1+\epsilon}$ gives the estimates $\hat{m}_{\Gamma}(t), m_{\Gamma}(t) \ll t^{1+\epsilon}$. Thus we get

$$
\left|\hat{\pi}_{\Gamma}^{(k)}(x ; \mathbf{r})-\pi_{\Gamma}^{(k)}(x ; \mathbf{r})\right| \ll \sum_{1 \leq i \leq k} \sum_{\substack{\operatorname{Prim}_{(}(\Gamma), j \geq 2 \\ t_{\gamma^{j}}<x}} t^{k-1+\epsilon} \frac{1}{j} \ll x^{k+1 / 2+\epsilon} .
$$

The claim follows immediately.

\subsection{Approximation of $I_{\Gamma}(t)$ by periodic functions}

In this subsection, we study $I_{\Gamma}$ in the view of the theory on arithmetic functions [30].

For an integer $q \geq 1$ and a function $f: \mathbb{N} \rightarrow \mathbb{C}$, define the semi-norm

$$
\|f\|_{q}:=\left(\limsup _{x \rightarrow \infty} \frac{1}{x} \sum_{1 \leq n \leq x}|f(n)|^{q}\right)^{1 / q} .
$$

The function $f$ is called a $q$-limit periodic function if, for any $\epsilon>0$, there is a periodic function $h$ such that $\|f-h\|_{q}<\epsilon$. The set $\mathcal{D}^{q}$ of all $q$-limit periodic functions becomes a Banach space with the norm $\|*\|_{q}$ if functions $f_{1}, f_{2}$ with $\left\|f_{1}-f_{2}\right\|_{q}=0$ are identified.

We now prove the following proposition.

Proposition 3.2. Let $q \geq 1$ be an integer and $f_{1}, \cdots, f_{q} \in \mathcal{D}^{q}$. Suppose that $f_{i}$ is approximated by a series of periodic functions $\left\{f_{i j}\right\}_{j \geq 1}$, namely $\left\|f_{i j}-f_{i}\right\|_{q} \rightarrow 0$ as $j \rightarrow \infty$. Without loss of generality, suppose that $f_{1 j}, \cdots, f_{q j}$ have the same period $N_{j}$ and $N_{j} \rightarrow \infty$ as $j \rightarrow \infty$. Then we have

$$
\lim _{x \rightarrow \infty} \frac{1}{x} \sum_{1 \leq t \leq x} f_{1}(t) \cdots f_{q}(t)=\lim _{j \rightarrow \infty} N_{j}^{q-1} \sum_{m \in \mathbb{Z}_{N_{j}}} F_{1 j}\left(m ; N_{j}\right) \cdots F_{q j}\left(m ; N_{j}\right),
$$

where

$$
F_{i j}\left(m ; N_{j}\right):=\lim _{x \rightarrow \infty} \frac{1}{x} \sum_{\substack{1 \leq t \leq x \\ t \equiv m \bmod N_{j}}} f_{i j}(t)=\frac{1}{N_{j}} f_{i j}(m)
$$


Proof. Let $q, j \geq 1$ be integers, $x>0$ a number sufficiently larger than $N_{j}$ and

$$
G(x):=\frac{1}{x} \sum_{1 \leq t \leq x} f_{1}(t) \cdots f_{q}(t), \quad G_{j}(x):=\frac{1}{x} \sum_{1 \leq t \leq x} f_{1 j}(t) \cdots f_{q j}(t) .
$$

Since $f_{1 j}, \cdots, f_{q j}$ are $N_{j}$-periodic, we have

$$
\begin{aligned}
G_{j}(x) & =\frac{1}{x} \sum_{0 \leq l \leq x / N_{j}} \sum_{0 \leq m<N_{j}} f_{1 j}\left(N_{j} l+m\right) \cdots f_{q j}\left(N_{j} l+m\right)+\frac{1}{x} \sum_{N_{j}\left[x / N_{j}\right] \leq t \leq x} f_{1 j}(t) \cdots f_{q j}(t) \\
& \rightarrow N_{j}^{q-1} \sum_{0 \leq m<N_{j}} F_{1 j}\left(m ; N_{j}\right) \cdots F_{q j}\left(m ; N_{j}\right) \quad \text { as } \quad x \rightarrow \infty .
\end{aligned}
$$

The assumption $\left\|f_{i}-f_{i j}\right\|_{q} \rightarrow 0$ as $j \rightarrow \infty$ gives that

$$
\begin{aligned}
& \limsup _{x \rightarrow \infty}\left|G(x)-G_{j}(x)\right| \leq\left\|f_{1} \cdots f_{q}-f_{1 j} \cdots f_{q j}\right\|_{1} \\
\leq & \sum_{1 \leq l \leq q}\left\|f_{1}\right\|_{q} \cdots\left\|f_{l-1}\right\|\left\|_{q}\right\| f_{l}-f_{l j}\left\|_{q}\right\| f_{l+1, j}\left\|_{q} \cdots\right\| f_{q j} \|_{q} \rightarrow 0 \quad \text { as } \quad j \rightarrow \infty .
\end{aligned}
$$

Since $\lim _{x \rightarrow \infty} G_{j}(x)$ exists for any $j$ and $G(x)$ does not depend on $j$, the clam in this proposition follows from (3.2) and (3.3).

Next, study the $q$-limit periodicity of $I_{\Gamma}$. By the definition of $I_{\Gamma}$ and the class number formula, we have

$$
I_{\Gamma}(t)=\sum_{u \in U(t)} \omega_{\Gamma}(t, u) u^{-1} L\left(1, D_{t, u}\right)
$$

where $L\left(1, D_{t, u}\right):=\prod_{p}\left(1-\left(D_{t, u} / p\right) p^{-1}\right)^{-1}$. For integers $P \geq 2$ and $M \geq 1$, let

$$
\beta_{\Gamma, P, M}(t):=\sum_{\substack{u \in U(t) \\ p \mid u \Rightarrow p \leq P \\ \operatorname{ord}_{p} u \leq M}} \omega_{\Gamma}(t, u) u^{-1} \prod_{p \leq P}\left(1-\left(D_{t, u} / p\right) p^{-1}\right)^{-1},
$$

and $\beta_{\Gamma, P}(t):=\lim _{M \rightarrow \infty} \beta_{\Gamma, P, M}(t)$. For $\Gamma=\mathrm{SL}_{2}(\mathbb{Z})$, we see that $\beta_{\Gamma, P, M}(t)$ is of $B_{P, M}:=$ $2^{2 M+3} \prod_{2<p \leq P} p^{2 M+1}$-periodic. Peter [23] approximated $I_{\mathrm{SL}_{2}(\mathbb{Z})}$ by $\beta_{\mathrm{SL}_{2}(\mathbb{Z}), P}, \beta_{\mathrm{SL}_{2}(\mathbb{Z}), P, M}$, and proved that $I_{\mathrm{SL}_{2}(\mathbb{Z})} \in \mathcal{D}^{q}$ for any $q \geq 1$.

For a congruence subgroup $\Gamma$ of level $n$, we see that $\beta_{\Gamma, P, M}(t)$ is of $n^{2} B_{P, M}$-periodic since $\omega_{\Gamma}(t, u)$ is a character on $\operatorname{PSL}_{2}\left(\mathbb{Z}_{n}\right)$. We prove in the following lemma, due to Peter's work [23], that $I_{\Gamma} \in \mathcal{D}^{q}$ for any congruence subgroup $\Gamma$ and any $q \geq 1$

Lemma 3.3. For any $q \geq 1$, there exists a constant $\epsilon>0$ such that

$$
\left\|I_{\Gamma}-\beta_{\Gamma, P, M}\right\|_{q} \ll P^{-\epsilon}+2^{-M}(\log P)^{2} \quad \text { as } \quad P, M \rightarrow \infty,
$$

where the implied constant depends on $q$ and $\Gamma$.

Proof. By the definition of $\beta_{\Gamma, P, M}(t)$ and $\beta_{\Gamma, P}(t)$, we have

$$
\left|\beta_{\Gamma, P}(t)-\beta_{\Gamma, P, M}(t)\right|=\sum_{\substack{u \in U(t) \\ p \mid u \Rightarrow p \leq P \\ \operatorname{ord}_{p} u \geq M(\exists p)}} \omega_{\Gamma}(t, u) u^{-1} \prod_{p \leq P}\left(1-\left(D_{t, u} / p\right) p^{-1}\right)^{-1} .
$$

Since $0 \leq \omega_{\Gamma}(t, u) \leq\left[\mathrm{SL}_{2}(\mathbb{Z}) ; \Gamma\right]$, the difference above is bounded by

$$
\left|\beta_{\Gamma, P}(t)-\beta_{\Gamma, P, M}(t)\right| \leq\left[\mathrm{SL}_{2}(\mathbb{Z}): \Gamma\right] \sum_{2 \leq p \leq P} \sum_{u \in U(t), p^{M} \mid u} u^{-1} \prod_{p_{1} \leq P}\left(1-\left(D_{t, u} / p_{1}\right) p_{1}^{-1}\right)^{-1}
$$


multiplicities in length spectra for congruence subgroups

$$
\ll(\log P)^{2} \sum_{2 \leq p \leq P} p^{-M} \ll 2^{-M}(\log P)^{2} .
$$

By virtue of Lemma 3.1 and Proposition 3.7 in [23] (see also Corollary 4.2) and (3.4), we obtain

$$
\begin{aligned}
\left\|I_{\Gamma}-\beta_{\Gamma, P, M}\right\|_{q} & \leq\left\|I_{\Gamma}-\beta_{\Gamma, P}\right\|_{q}+\left\|\beta_{\Gamma, P}-\beta_{\Gamma, P, M}\right\|_{q} \\
& \leq\left[\mathrm{SL}_{2}(\mathbb{Z}): \Gamma\right]\left\|I_{\mathrm{SL}_{2}(\mathbb{Z})}-\beta_{\mathrm{SL}_{2}(\mathbb{Z}), P}\right\|_{q}+\sup _{t \geq 3}\left|\beta_{\Gamma, P}(t)-\beta_{\Gamma, P, M}(t)\right|_{q} \\
& \ll P^{-\epsilon}+2^{-M}(\log P)^{2}
\end{aligned}
$$

for some $\epsilon>0$.

\subsection{Partial sum of multiplicities}

In this subsection, we study the growth of partial sums of $I_{\Gamma}(t)$ to give the expression of $c_{\Gamma}^{(k)}(\mathbf{r})$ in Theorem 3.1, First we prepare the following variation of the prime geodesic theorem called the Tchebotarev type prime geodesic theorem.

Theorem 3.4. (Tchebotarev type prime geodesic theorem, [27] and [32]) Let $\Gamma_{1}, \Gamma_{2}$ be discrete subgroups of $\mathrm{SL}_{2}(\mathbb{R})$ with $\operatorname{vol}\left(\Gamma_{1} \backslash H\right)<\infty, \Gamma_{1} \triangleright \Gamma_{2},\left[\Gamma_{1}: \Gamma_{2}\right]<\infty$. Then, for $[g] \in \operatorname{Conj}\left(\Gamma_{2} \backslash \Gamma_{1}\right)$, we have

$$
\#\left\{\gamma \in \operatorname{Prim}\left(\Gamma_{1}\right) \mid \sigma(\gamma) \subset[g], N(\gamma)<x\right\} \sim \frac{\#[g]}{\# \Gamma_{2} \backslash \Gamma_{1}} \operatorname{li}(x),
$$

where $\sigma: \Gamma_{1} \rightarrow \Gamma_{2} \backslash \Gamma_{1}$ is a projection.

Due to Theorem 3.5, we get the following lemma.

Lemma 3.5. Let $N \geq 1$ be an integer, $m \in \mathbb{Z}_{N}$ and $\Gamma$ a congruence subgroup of $\mathrm{SL}_{2}(\mathbb{Z})$. Then we have

$$
\lim _{x \rightarrow \infty} \frac{1}{x} \sum_{\substack{3 \leq t \leq x \\ t \equiv m \bmod N}} I_{\Gamma}(t)=\frac{\#\{\gamma \in \Gamma(N) \backslash \Gamma \Gamma(N) \mid \operatorname{tr} \gamma \equiv m \bmod N\}}{\# \Gamma(N) \backslash \Gamma \Gamma(N)}
$$

Proof. It is easy to see that $\Gamma_{1}=\Gamma$ and $\Gamma_{2}=\Gamma \cap \hat{\Gamma}(N)$ satisfy the condition in Theorem 3.4 . Since $\operatorname{tr} \gamma_{1} \equiv \alpha \operatorname{tr} \gamma_{2} \bmod N$ for some $\alpha^{2} \equiv 1 \bmod N$ if $\gamma_{1} \sim \gamma_{2}$ in $\Gamma \cap \hat{\Gamma}(N) \backslash \Gamma$, we have

$$
\lim _{x \rightarrow \infty} \frac{1}{x} \sum_{\substack{3 \leq t \leq x \\ t=\alpha m \bmod N \\ \alpha^{2} \equiv 1 \bmod N}} I_{\Gamma}(t)=\frac{\#\left\{\gamma \in \Gamma \cap \hat{\Gamma}(N) \backslash \Gamma \mid \operatorname{tr} \gamma \equiv \alpha m \bmod N, \alpha^{2} \equiv 1 \bmod N\right\}}{\# \Gamma \cap \hat{\Gamma}(N) \backslash \Gamma} .
$$

Due to the isomorphism theorem, we have

$$
\Gamma \cap \hat{\Gamma}(N) \backslash \Gamma \simeq \hat{\Gamma}(N) \backslash \Gamma \hat{\Gamma}(N)
$$

with a one-to-one correspondence $(\Gamma \cap \hat{\Gamma}(N)) \gamma \longleftrightarrow \hat{\Gamma}(N) \gamma(\gamma \in \Gamma)$. Thus the equation (3.6) is written by

$$
\lim _{x \rightarrow \infty} \frac{1}{x} \sum_{\substack{3 \leq t \leq x \\ t \equiv \alpha m \bmod N \\ \alpha^{2} \equiv 1 \bmod N}} I_{\Gamma}(t)=\frac{\#\left\{\gamma \in \hat{\Gamma}(N) \backslash \Gamma \hat{\Gamma}(N) \mid \operatorname{tr} \gamma \equiv \alpha m \bmod N, \alpha^{2} \equiv 1 \bmod N\right\}}{\# \hat{\Gamma}(N) \backslash \Gamma \hat{\Gamma}(N)} .
$$


According to Lemma 2.11 and 2.19 in [25], we see that

$$
A(m ; N ; u):=\lim _{x \rightarrow \infty} \frac{1}{x} \sum_{\substack{t<x, t \equiv m \bmod N \\ D_{t, u} \in \mathcal{D}}} u^{-1} L\left(1, D_{t, u}\right)
$$

satisfies that $A(m ; N, u)=A(\alpha m ; N, u)$ for any $u \geq 1, m \in \mathbb{Z}_{N}, \alpha^{2} \equiv 1 \bmod N$. Furthermore, since $\omega_{\Gamma}(t, u)$ is a character on $\operatorname{PSL}_{2}\left(\mathbb{Z}_{n}\right)$, taking the sum of $A(m ; N ; u)$ over $u$, we get the equation in Lemma 3.5 from (3.7).

The following lemma describes the values in Proposition 3.5 for $\Gamma \Gamma\left(p^{r}\right)=\mathrm{SL}_{2}(\mathbb{Z})$.

Lemma 3.6. When $p=2$ and $r \geq 6$, we have

$$
\begin{aligned}
& \#\left\{\gamma \in \mathrm{SL}_{2}\left(\mathbb{Z}_{2^{r}}\right) \mid \operatorname{tr} \gamma \equiv t \bmod 2^{r}\right\} \\
& = \begin{cases}2^{2 r-1} & (2 \nmid t), \\
3 \cdot 2^{2 r-2}, & (4 \mid t), \\
3 \cdot 2^{2 r-1}, & \left(t \equiv 16 t_{1} \pm 2 \bmod 2^{r}, t_{1} \equiv 5 \bmod 8,\right. \\
& \text { or } \left.t \equiv 2^{l} t_{1} \pm 2 \bmod 2^{r}, t_{1} \equiv 1 \bmod 8, l \geq 6: \text { even }\right), \\
5 \cdot 2^{2 r-2}, & \left(t \equiv 16 t_{1} \pm 2 \bmod 2^{r}, t_{1} \not \equiv 5 \bmod 8\right), \\
3 \cdot 2^{2 r-1}-2^{2 r-l / 2}, & \left(t \equiv 2^{l} t_{1} \pm 2 \bmod 2^{r}, t_{1} \not \equiv 1 \bmod 8, l \geq 6: \text { even }\right), \\
3 \cdot\left(2^{2 r-1}-2^{2 r-(l+3) / 2}\right), & \left(t \equiv 2^{l} t_{1} \pm 2 \bmod 2^{r}, 2 \nmid t_{1}, l \geq 3: \text { odd }\right), \\
3 \cdot 2^{2 r-1}-2^{\lfloor(3 r-1) / 2\rfloor}, & \left(t \equiv \pm 2 \bmod 2^{r}\right) .\end{cases}
\end{aligned}
$$

When $p \geq 3$ and $r \geq 1$, we have

$$
\#\left\{\gamma \in \mathrm{SL}_{2}\left(\mathbb{Z}_{p^{r}}\right) \mid \operatorname{tr} \gamma \equiv t \bmod p^{r}\right\}
$$

$$
= \begin{cases}p^{2 r-1}(p-1), & ((T / p)=-1), \\ p^{2 r-1}(p+1), & \left((T / p)=1 \text { or } p^{l} \| T, 2 \mid l,\left(\frac{T}{p^{l}} / p\right)=1\right), \\ p^{2 r}+p^{2 r-1}-2 p^{2 r-l / 2-1}, & \left(p^{l} \| T, 2 \mid l,\left(\frac{T}{p^{l}} / p\right)=-1\right), \\ p^{2 r}+p^{2 r-1}-p^{2 r-(l+1) / 2}-p^{2 r-(l+3) / 2}, & \left(p^{l} \| T, 2 \nmid l\right), \\ p^{2 r}+p^{2 r-1}-p^{\lfloor(3 r-1) / 2\rfloor}, & \left(T \equiv 0 \bmod p^{r}\right),\end{cases}
$$

where $T:=t^{2}-4$.

Proof. For simplicity, we prove only for $p \geq 3$. Let $\gamma:=\left(\begin{array}{ll}a & b \\ c & d\end{array}\right) \in \operatorname{SL}_{2}(\mathbb{Z})$. Since $\operatorname{tr} \gamma=$ $a+d \equiv t$ and $a d-b c \equiv 1, \#\left\{\gamma \in \mathrm{SL}_{2}\left(\mathbb{Z}_{p^{r}}\right) \mid \operatorname{tr} \gamma \equiv t \bmod p^{r}\right\}$ is the number of $a, b, c \in \mathbb{Z}_{p^{r}}$ with

$$
b c \equiv T / 4-(a-t / 2)^{2} \bmod p^{r} .
$$

(i) The case of $(T / p)=-1$. In this case, there are no $a$ such that $b c=T / 4-(a-t / 2)^{2}$ is divided by $p$. Then $c$ is uniquely determined by $c \equiv b^{-1}\left(T / 4-(a-t / 2)^{2}\right)$ for given $a \in \mathbb{Z}_{p^{r}}$ and $b \in \mathbb{Z}_{p^{r}}^{*}$. Thus the number of such $(a, b, c)$ is $p^{r} \cdot p^{r-1}(p-1)=p^{2 r-1}(p-1)$.

(ii) The case of $(T / p)=1$. The number of $a$ with $p \nmid b c$ is $p^{r-1}(p-2)$ and, for such $a$ and given $b \in \mathbb{Z}_{p^{r}}^{*}, c$ is uniquely determined by $c \equiv b^{-1}\left(T / 4-(a-t / 2)^{2}\right)$. Then the number of such $(a, b, c)$ is $p^{2 r-2}(p-1)(p-2)$.

The number of $a$ with $p^{l} \| b c(1 \leq l \leq r-1)$ is $2 p^{r-l-1}(p-1)$. In this case, $b \equiv b_{1} p^{l_{1}}$ and $c \equiv c_{1} p^{l-l_{1}}$ for $0 \leq l_{1} \leq l, b_{1} \in \mathbb{Z}_{p^{r-l_{1}}}^{*}$ and $c_{1} \in \mathbb{Z}_{p^{r-l+l_{1}}}^{*}$. Then the number of $b_{1}$, $c_{1}$ for given 
$a$ and $l_{1}$ is $p^{r-l_{1}-1}(p-1) \cdot p^{l_{1}}=p^{r-1}(p-1)$.

The number of $a$ with $b c \equiv 0$ is 2 . In this case, $b \equiv b_{1} p^{l_{1}}$ and $c \equiv c_{1}^{l_{2}}$ for $0 \leq l_{1}, l_{2} \leq r$, $l_{1}+l_{2} \geq r, b_{1} \in \mathbb{Z}_{p^{r-l_{1}}}^{*}$ and $c_{1} \in \mathbb{Z}_{p^{r-l_{2}}}^{*}$.

Thus the total number of $(a, b, c)$ is

$$
\begin{aligned}
p^{2 r-2}(p-1)(p-2) & +\sum_{1 \leq l \leq r-1} \sum_{0 \leq l_{1} \leq l} 2 p^{2 r-l-2}(p-1)^{2} \\
& +\sum_{0 \leq l_{1} \leq r} \sum_{r-l_{1} \leq l_{2} \leq r} 2 \varphi\left(p^{r-l_{1}}\right) \varphi\left(p^{r-l_{2}}\right)=p^{2 r}+p^{2 r-1},
\end{aligned}
$$

where $\varphi(n):=\# \mathbb{Z}_{n}^{*}$.

(iii) The case of $p \mid T$. Suppose that $p^{l} \| T(1 \leq l \leq r)$. If $p^{s} \| a$ for $0 \leq 2 s<l$, then $p^{2 s} \| b c$. In this case, $b \equiv b_{1} p^{l_{1}}$ and $c \equiv c_{1} p^{2 s-l_{1}}$ for $0 \leq l_{1} \leq 2 s, b_{1} \in \mathbb{Z}_{p^{r-l_{1}}}^{*}$ and $c_{1} \in \mathbb{Z}_{p^{r-2 s+l_{1}}}^{*}$. Then the number of $(b, c)$ for given $a$ and $l_{1}$ is $p^{r-1}(p-1)$.

The number of $(b, c)$ for $p^{s} \mid a$ and $2 s \geq l$ is different between the case of $2 \mid l,\left(\frac{T}{p^{l}} / p\right)=1$ and otherwise. In the later case, there are no $a$ with $p^{l+1} \mid b c$. Then the number of $(a, b, c)$ is

$$
\begin{aligned}
& \sum_{0 \leq s<l / 2} \sum_{0 \leq l_{1} \leq 2 s} p^{2 r-s-2}(p-1)^{2}+\sum_{0 \leq l_{1} \leq l} p^{r-\lfloor(l+1) / 2\rfloor} p^{r-1}(p-1) \\
= & \begin{cases}p^{2 r}+p^{2 r-1}-2 p^{2 r-l / 2-1}, & \left(p^{l} \| T, 2 \mid l,\left(\frac{T}{p^{l}} / p\right)=-1\right), \\
p^{2 r}+p^{2 r-1}-p^{2 r-(l+1) / 2}-p^{2 r-(l+3) / 2}, & \left(p^{l} \| T, 2 \nmid l\right) .\end{cases}
\end{aligned}
$$

In the former case, there are $a$ with $p^{l+1} \mid b c$. The number of $a$ with $p^{l+s} \| b c$ is $p^{r-l / 2-1}(p-2)$ when $s=0,2 p^{r-l / 2-s-1}(p-1)$ when $1 \leq s \leq r-l-1$ and 2 when $s=r-l$. Since the number of $(b, c)$ are given similar to the case (ii), we can calculate the number of $(a, b, c)$ as follows.

$$
\begin{aligned}
& \sum_{0 \leq s<l / 2} \sum_{0 \leq l_{1} \leq 2 s} p^{2 r-s-2}(p-1)^{2} \sum_{0 \leq l_{1} \leq l} p^{2 r-l / 2-2}(p-1)(p-2) \\
& +\sum_{\substack{1 \leq s \leq r-l-1 \\
0 \leq l_{1} \leq l+s}} 2 p^{2 r-l / 2-s-2}(p-1)^{2}+\sum_{0 \leq l_{1} \leq r} \sum_{r-l_{1} \leq l_{2} \leq r} 2 \varphi\left(p^{r-l_{1}}\right) \varphi\left(p^{r-l_{2}}\right) \\
= & p^{2 r}+p^{2 r-1} .
\end{aligned}
$$

The number of $(a, b, c)$ in the case of $p^{r} \mid T$ is computed similarly.

\subsection{Proof of Theorem 3.1}

According to Lemma 3.3, we see that $I_{\Gamma} \in \mathcal{D}^{q}$ for any $q \geq 1$ and $I_{\Gamma}$ is approximated by $\beta_{\Gamma, P, M}$ as $M, P \rightarrow \infty$ with $M>4 \log \log P$. Then Proposition 3.2 holds for $f_{i}(t)=I_{\Gamma}\left(t+r_{i}\right)$ and $\left\{f_{i j}(t)\right\}_{j}=\left\{\beta_{\Gamma, P, M}\left(t+r_{i}\right)\right\}_{P, M}$ with the periods $\left\{N_{j}\right\}_{j}=\left\{n^{2} B_{P, M}\right\}_{P, M}$. Thus we have

$$
c_{\Gamma}^{(k)}(\mathbf{r}, j):=N_{j}^{k-1} \sum_{m \in \mathbb{Z}_{N_{j}}} F_{1 j}\left(m ; N_{j}\right) \cdots F_{k j}\left(m ; N_{j}\right) \rightarrow c_{\Gamma}^{(k)}(\mathbf{r}) \quad \text { as } \quad j \rightarrow \infty .
$$

We now compare $c_{\Gamma}^{(k)}(\mathbf{r}, j)$ and

$$
\tilde{c}_{\Gamma}^{(k)}(\mathbf{r}, j):=N_{j}^{k-1} \sum_{m \in \mathbb{Z}_{N_{j}}} F_{1}\left(m ; N_{j}\right) \cdots F_{k}\left(m ; N_{j}\right)
$$


where $F_{i}\left(m ; N_{j}\right):=F_{\Gamma}\left(m+r_{i} ; N_{j}\right)$ is given in Theorem 3.1. The difference is bounded by

$$
\begin{aligned}
\left|c_{\Gamma}^{(k)}(\mathbf{r}, j)-\tilde{c}_{\Gamma}^{(k)}(\mathbf{r}, j)\right| \leq & N_{j}^{k-1} \sum_{m \in \mathbb{Z}_{N_{j}}} \sum_{1 \leq l \leq k} F_{1 j}\left(m ; N_{j}\right) \cdots F_{l-1, j}\left(m ; N_{j}\right) \\
& \times\left|F_{l j}\left(m ; N_{j}\right)-F_{l}\left(m ; N_{j}\right)\right| F_{l+1}\left(m ; N_{j}\right) \cdots F_{k}\left(m ; N_{j}\right) .
\end{aligned}
$$

By the definition of $F_{i j}$ and $\beta_{\Gamma, P, M}$, we have

$$
\begin{aligned}
N_{j} F_{i j}\left(m ; N_{j}\right) & =\beta_{\Gamma, P, M}\left(t+r_{j}\right) \\
& \leq\left[\mathrm{SL}_{2}(\mathbb{Z}): \Gamma\right]\left(\prod_{p_{1} \leq P} \sum_{0 \leq l \leq M} p_{1}^{-l}\right) \sum_{p_{2} \leq P}\left(1-p_{2}^{-1}\right)^{-1} \ll(\log P)^{2} .
\end{aligned}
$$

For $F_{i}$, since

$$
F_{i}\left(m ; n_{1} n_{2}\right)=F_{i}\left(m ; n_{1}\right) F_{i}\left(m ; n_{2}\right)
$$

for relatively prime $n_{1}$ and $n_{2}$ and $\Gamma \hat{\Gamma}(N)=\mathrm{SL}_{2}(\mathbb{Z})$ except a finite number of $N$, we see that

$$
N_{j} F_{i}\left(m ; N_{j}\right) \ll \prod_{p \leq P} \frac{p^{2 M+1} \#\left\{\gamma \in \mathrm{SL}_{2}\left(\mathbb{Z}_{p^{2 M+1}}\right) \mid \operatorname{tr} \equiv m+r_{i} \bmod p^{2 M+1}\right\}}{\# \mathrm{SL}_{2}\left(\mathbb{Z}_{p^{2 M+1}}\right)} .
$$

Due to Lemma 3.6 and $\# \mathrm{SL}_{2}\left(\mathbb{Z}_{p^{r}}\right)=p^{3 r-2}\left(p^{2}-1\right)$, we have

$$
N_{j} F_{i}\left(m ; N_{j}\right) \ll \prod_{p \leq P} \frac{p^{2 M+1} p^{4 M+1}(p+1)}{p^{6 M+1}\left(p^{2}-1\right)}=\prod_{p \leq P}\left(1-p^{-1}\right)^{-1} \ll \log P .
$$

Applying (3.11), (3.13) and Lemma 3.3 into (3.10), we obtain

$$
\begin{aligned}
\left|c_{\Gamma}^{(k)}(\mathbf{r}, j)-\tilde{c}_{\Gamma}^{(k)}(\mathbf{r}, j)\right| & \ll(\log P)^{2 k-2} \sum_{1 \leq l \leq k} \sum_{m \in \mathbb{Z}_{N_{j}}}\left|F_{l j}\left(m ; N_{j}\right)-F_{l}\left(m ; N_{j}\right)\right| \\
& \leq(\log P)^{2 k-2} \sum_{1 \leq l \leq k}\left\|f_{l j}-f_{l}\right\|_{1} \\
& \ll(\log P)^{2 k-2}\left(P^{-\epsilon}+2^{-M}(\log P)^{2}\right) \rightarrow 0
\end{aligned}
$$

as $P, M \rightarrow \infty$ with $M \gg \log P$, and then $c_{\Gamma}^{(k)}(\mathbf{r})=\lim _{j \rightarrow \infty} \tilde{c}_{\Gamma}^{(k)}(\mathbf{r}, j)$. Thus the expression of $c_{\Gamma}^{(k)}(\mathbf{r})$ as a product over $p$ follows from the multiplicative property (3.12) of $F_{i}$.

\section{Examples}

In this section, we calculate $c_{\Gamma}^{(k)}(0)$ for $\Gamma=\mathrm{SL}_{2}(\mathbb{Z}), \Gamma_{0}(n), \hat{\Gamma}(n)$.

\subsection{The case of $\Gamma=\mathrm{SL}_{2}(\mathbb{Z})$}

In this case, it is clear that $\Gamma \Gamma\left(p^{l}\right)=\mathrm{SL}_{2}(\mathbb{Z})$ and $\# \mathrm{SL}_{2}\left(\mathbb{Z}_{p^{r}}\right)=p^{3 r-2}\left(p^{2}-1\right)$. We now propose the following lemma.

Lemma 4.1. Let $p^{r}$ be a power of prime and $1 \leq l \leq r-1$. Then we have

$$
\begin{aligned}
\#\left\{t \in \mathbb{Z}_{p^{r}} \mid(T / p)=1\right\} & =p^{r-1}(p-3) / 2, \\
\#\left\{t \in \mathbb{Z}_{p^{r}} \mid(T / p)=-1\right\} & =p^{r-1}(p-1) / 2, \\
\#\left\{t \in \mathbb{Z}_{p^{r}}\left|p^{l}\right| \mid T,\left(T p^{-l} / p\right)=1\right\} & =p^{r-1}(p-1),
\end{aligned}
$$


multiplicities in length spectra for congruence subgroups

$$
\begin{gathered}
\#\left\{t \in \mathbb{Z}_{p^{r}}\left|p^{l}\right| \mid T,\left(T p^{-l} / p\right)=-1\right\}=p^{r-1}(p-1), \\
\#\left\{t \in \mathbb{Z}_{p^{r}} \mid T \equiv 0 \bmod p^{r}\right\}=2 .
\end{gathered}
$$

Proof. Let $\alpha \in \mathbb{Z}_{p^{r}}^{*}-\{ \pm 1\}$ and $t(\alpha):=\alpha+\alpha^{-1} \bmod p$. Then $t(\alpha)^{2}-4=\left(\alpha-\alpha^{-1}\right)^{2}$ is a quadratic residue of $p$. Conversely, suppose that $t \in \mathbb{Z}_{p}$ satisfies $(T / p)=1$ and $\alpha(t):=$ $\left(t+\sqrt{t^{2}-4}\right) / 2$. Then we see that $\alpha(t) \in\left(\mathbb{Z}_{p}\right)^{*}-\{ \pm 1\}$ and $\alpha(t)+\alpha(t)^{-1}=t$. Thus we have

$$
\#\left\{t \in \mathbb{Z}_{p} \mid(T / p)=1\right\}=\#\left\{\alpha \in \mathbb{Z}_{p}^{*}-\{ \pm 1\}\right\} / 2=(p-3) / 2
$$

and the result for $(T / p)=1$ follows immediately. Since

$$
\#\left\{t \in \mathbb{Z}_{p^{r}}|p| T\right\}=\#\left\{t \in \mathbb{Z}_{p^{r}} \mid t \equiv \pm 2 \bmod p\right\}=2 p^{r-1},
$$

we also get the result for $(T / p)=-1$.

If $p^{l} \| T$ then $t= \pm 2+t_{1} p^{l}$ for $t_{1} \in \mathbb{Z}_{p^{r-l}}^{*}$ and $T / p^{l}=t_{1}^{2} p \pm 4 t_{1} \equiv \pm 4 t_{1} \bmod p$. Thus the results for $p^{l} \| T$ is obtained easily.

Combining Lemma 3.6 and 4.1, we get

$$
\begin{aligned}
\lim _{r \rightarrow \infty} 2^{r(k-1)} \sum_{m \in \mathbb{Z}_{2^{r}}} F_{\mathrm{SL}_{2}(\mathbb{Z})}\left(m ; 2^{r}\right)= & \frac{1}{2}\left(\frac{2}{3}\right)^{k}+\frac{1}{4}+2^{k-6}+\frac{3}{64}\left(\frac{5}{3}\right)^{k}+\sum_{\substack{3 \leq l \leq r-1 \\
\text { odd }}} 2^{k-l}\left(1-2^{-(l+1) / 2}\right)^{k} \\
& +\sum_{\substack{6 \leq l \leq r-1 \\
\text { even }}} 2^{k-l-2}\left(1-3\left(1-3^{-1} \cdot 2^{-l / 2+1}\right)^{k}\right),
\end{aligned}
$$

and, for $p \geq 3$,

$$
\begin{aligned}
& \lim _{r \rightarrow \infty} p^{r(k-1)} \sum_{m \in \mathbb{Z}_{p^{r}}} F_{\mathrm{SL}_{2}(\mathbb{Z})}\left(m ; p^{r}\right) \\
= & \frac{1}{2}\left(1-3 p^{-1}\right)\left(1+p^{-1}\right)^{-k}+\frac{1}{2}\left(1-p^{-1}\right)^{-k+1}+p^{-2}\left(1+p^{-1}\right)\left(1-p^{-1}\right)^{-k} \\
& +\sum_{l \geq 1} 2 p^{-2 l+1}\left(1-p^{-1}\right)^{-k+1}\left(1-p^{-l}\right)^{k}+\sum_{l \geq 1} p^{-2 l}\left(1-p^{-1}\right)\left(1-p^{-2}\right)^{-k}\left(1+p^{-1}-2 p^{-l-1}\right)^{k} .
\end{aligned}
$$

According to Theorem 3.1 , we see that $c_{\mathrm{SL}_{2}(\mathbb{Z})}^{(k)}(0)$ is the product of these values over primes $p$. Especially, for $k=2,3$, we have

$$
\begin{aligned}
& c_{\mathrm{SL}_{2}(\mathbb{Z})}^{(2)}(0)=\frac{1015}{864} \prod_{p \geq 3} \frac{p^{2}\left(p^{3}+p^{2}-p-3\right)}{(p-1)^{2}(p+1)^{3}}, \\
& c_{\mathrm{SL}_{2}(\mathbb{Z})}^{(3)}(0)=\frac{682495}{428544} \prod_{p \geq 3} \frac{p^{8}+p^{7}+p^{6}-5 p^{5}-5 p^{3}-5 p^{2}-p-1}{(p-1)^{2}(p+1)^{2}\left(p^{4}+p^{3}+p^{2}+p+1\right)} .
\end{aligned}
$$

Note that $c_{\mathrm{SL}_{2}(\mathbb{Z})}^{(2)}(0)$ coincides with the coefficient given in $[3,23]$.

\subsection{The case of $\Gamma=\Gamma_{0}(n)$}

Let $n$ be an odd integer and $\Gamma_{0}(n)$ be a congruence subgroup of $\mathrm{SL}_{2}(\mathbb{Z})$ consisting of the elements $\gamma$ with $n \mid \gamma_{21}$. We see that $\Gamma \Gamma\left(p^{r}\right)=\Gamma_{0}\left(p^{\min \left(r, \operatorname{ord}_{p} n\right)}\right)$ for $p \mid n$ and $\Gamma \Gamma\left(p^{r}\right)=\mathrm{SL}_{2}(\mathbb{Z})$ for $p \nmid n$. Since $\left[\mathrm{SL}_{2}(\mathbb{Z}): \Gamma_{0}\left(p^{N}\right)\right]=p^{N-1}(p+1)$, we have $\# \Gamma_{0}\left(p^{N}\right) / \Gamma\left(p^{r}\right)=p^{3 r-N-1}(p-1)$ for $N \leq r$. The value $\#\left\{\gamma \in \Gamma_{0}\left(p^{N}\right) / \Gamma\left(p^{r}\right) \mid \operatorname{tr} \gamma \equiv t \bmod p^{r}\right\}$ is given as follows. 


\section{Lemma 4.2.}

$\#\left\{\gamma \in \Gamma_{0}\left(p^{N}\right) / \Gamma\left(p^{r}\right) \mid \operatorname{tr} \gamma \equiv t \bmod p^{r}\right\}$

$$
= \begin{cases}2 p^{2 r+l / 2-N-1}, & \left(p^{l} \| T, 0 \leq l \leq N, 2 \mid l,\left(\frac{T}{p^{l}} / p\right)=1\right), \\ p^{2 r-\lfloor(N+1) / 2\rfloor}+p^{2 r-\lfloor N / 2\rfloor-1}, & \left(p^{l} \| T, l \geq N, 2 \mid l,\left(\frac{T}{p^{l}} / p\right)=1\right), \\ p^{2 r-\lfloor(N+1) / 2\rfloor}+p^{2 r-\lfloor N / 2\rfloor-1}-2 p^{2 r-l / 2-1}, & \left(p^{l} \| T, l \geq N, 2 \mid l,\left(\frac{T}{p^{l}} / p\right)=-1\right), \\ p^{2 r-\lfloor(N+1) / 2\rfloor}+p^{2 r-\lfloor N / 2\rfloor-1}-p^{2 r-(l+1) / 2}-p^{2 r-(l+3) / 2}, & \left(p^{l} \| T, l \geq N, 2 \nmid l\right), \\ p^{2 r-\lfloor(N+1) / 2\rfloor}+p^{2 r-\lfloor N / 2\rfloor-1}-p^{\lfloor(3 r-1) / 2\rfloor}, & (T \equiv 0), \\ 0, & \text { (otherwise) } .\end{cases}
$$

Proof. The element $\gamma:=\left(\begin{array}{ll}a & b \\ c & d\end{array}\right) \in \Gamma_{0}\left(p^{N}\right)$ satisfies $p^{N} \mid c$. Then, picking up such $\gamma$ 's, we can calculate $\#\left\{\gamma \in \Gamma_{0}\left(p^{N}\right) / \Gamma\left(p^{r}\right) \mid \operatorname{tr} \gamma \equiv t \bmod p^{r}\right\}$ similar to the case of $\Gamma=\mathrm{SL}_{2}(\mathbb{Z})$.

Computing $c_{\Gamma_{0}(n)}^{(k)}(0)$ similar to the case of $\Gamma=\mathrm{SL}_{2}(\mathbb{Z})$, we get

where

$$
\begin{aligned}
c_{\Gamma_{0}(n)}^{(2)}(0)= & \frac{1015}{864} \prod_{p \geq 3, p \nmid n} \frac{p^{2}\left(p^{3}+p^{2}-p-3\right)}{(p-1)^{2}(p+1)^{3}} \prod_{p^{N} \| n} \frac{2 p\left(N p^{2}-p-N\right)}{(p-1)^{2}(p+1)}, \\
c_{\Gamma_{0}(n)}^{(3)}(0)= & \frac{682495}{428544} \prod_{p \geq 3, p \nmid n} \frac{p^{8}+p^{7}+p^{6}-5 p^{5}-5 p^{3}-5 p^{2}-p-1}{(p-1)^{2}(p+1)^{2}\left(p^{4}+p^{3}+p^{2}+p+1\right)}, \\
& \times \prod_{p^{N} \| n} \frac{p^{2}\left(p^{\lfloor(N-1) / 2\rfloor} h_{1}(p)-2 h_{2}(p)\right)}{(p-1)^{3}(p+1)\left(p^{4}+p^{3}+p^{2}+p+1\right)},
\end{aligned}
$$

$$
\begin{aligned}
& h_{1}(p):= \begin{cases}(p+1)\left(p^{6}+2 p^{5}+5 p^{4}+2 p^{3}+5 p^{2}+2 p+1\right), & (2 \mid N) \\
2\left(4 p^{6}+9 p^{5}+14 p^{4}+12 p^{3}+14 p^{2}+9 p+4\right), & (2 \nmid N),\end{cases} \\
& h_{2}(p):= \begin{cases}(p+1)^{2}\left(p^{4}+3 p^{3}+p^{2}+3 p+1\right), & (2 \mid N), \\
2(p+1)(p+3)\left(p^{4}+p^{3}+p^{2}+p+1\right), & (2 \nmid N) .\end{cases}
\end{aligned}
$$

Note that $c_{\Gamma_{0}(n)}^{(2)}(0)$ for square free $n$ coincides with the coefficient given in [17].

\subsection{The case of $\Gamma=\hat{\Gamma}(n)$}

Let $n$ be an odd integer. When $\Gamma=\hat{\Gamma}(n)$, we see that $\Gamma \Gamma\left(p^{r}\right)=\hat{\Gamma}\left(p^{\min \left(r, \operatorname{ord}_{p} n\right)}\right)$ for $p \mid n$ and

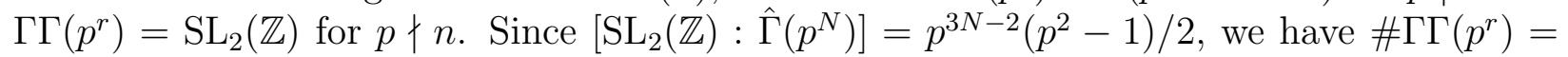
$2 p^{3(r-N)}$ for $N \geq r$. The value $\#\left\{\gamma \in \Gamma_{0}\left(p^{N}\right) / \Gamma\left(p^{r}\right) \mid \operatorname{tr} \gamma \equiv t \bmod p^{r}\right\}$ is given as follows.

\section{Lemma 4.3.}

$$
\#\left\{\gamma \in \hat{\Gamma}\left(p^{N}\right) / \Gamma\left(p^{r}\right) \mid \operatorname{tr} \gamma \equiv t \bmod p^{r}\right\}
$$




$$
= \begin{cases}p^{2 r-N-1}(p-1), & ((T / p)=-1), \\ p^{2 r-N-1}(p+1), & \left.\left((T / p)=1 \text { or } p^{l} \| T, 2 \mid l,\left(\frac{T}{p^{l}} / p\right)=1\right)\right), \\ p^{2 r-N}+p^{2 r-N-1}-2 p^{2 r-l / 2-1}, & \left(p^{l} \| T, 2 \mid l,\left(\frac{T}{p^{l}} / p\right)=-1\right), \\ p^{2 r-N}+p^{2 r-N-1}-p^{2 r-(l+1) / 2}-p^{2 r-(l+3) / 2}, & \left(p^{l} \| T, 2 \nmid l\right), \\ p^{2 r-N}+p^{2 r-N-1}-p^{\lfloor(3 r-1) / 2\rfloor}, & (T \equiv 0), \\ 0, & \left(t \neq \equiv \pm 2 \bmod p^{2 N}\right),\end{cases}
$$

where $T:=(t \pm 2) / p^{2 N}$ for $t \equiv \mp 2 \bmod p^{2 N}$.

Proof. The element $\gamma:=\left(\begin{array}{ll}a & b \\ c & d\end{array}\right) \in \hat{\Gamma}\left(p^{N}\right)$ satisfies $p^{N}\left|b, p^{N}\right| c, a \equiv d \equiv \pm 1 \bmod p^{N}$. Then, picking up such $\gamma$ 's, we can get the result similar to the case of $\Gamma=\mathrm{SL}_{2}(\mathbb{Z})$.

Computing $c_{\hat{\Gamma}(n)}^{(k)}(0)$ similar to the case of $\Gamma=\mathrm{SL}_{2}(\mathbb{Z})$, we get

$$
\begin{aligned}
c_{\hat{\Gamma}(n)}^{(2)}(0)= & \frac{1015}{864} \prod_{p \geq 3, p \nmid n} \frac{p^{2}\left(p^{3}+p^{2}-p-3\right)}{(p-1)^{2}(p+1)^{3}} \prod_{p^{N} \| n} \frac{p^{2 N-1}\left(p^{2}+p+1\right)}{2(p+1)}, \\
c_{\hat{\Gamma}(n)}^{(3)}(0)= & \frac{682495}{428544} \prod_{p \geq 3, p \nmid n} \frac{p^{8}+p^{7}+p^{6}-5 p^{5}-5 p^{3}-5 p^{2}-p-1}{(p-1)^{2}(p+1)^{2}\left(p^{4}+p^{3}+p^{2}+p+1\right)} \\
& \times \prod_{p^{N}} \frac{p^{4 N-2}\left(p^{6}+p^{5}+4 p^{4}+p^{3}+4 p^{2}+p+1\right)}{4\left(p^{4}+p^{3}+p^{2}+p+1\right)} .
\end{aligned}
$$

\section{References}

[1] R. Aurich and J. Marklof, Trace formulae for three-dimensional hyperbolic lattices and application to a strongly chaotic tetrahedral billiard, Phys. D 92 (1996), pp.101-129.

[2] E. Bogomolny, B. Georgeot, M.J. Giannoni and C. Schmit, Arithmetical chaos, Physics Reports 291 (1997), pp.219-324.

[3] E. Bogomolny, F. Leyvraz and C. Schmit, Distribution of eigenvalues for the modular group, Commun. Math. Phys. 176 (1996), pp.575-617.

[4] E. Bogomolny and C. Schmit, Multiplicities of periodic orbit lengths for non-arithmetic models, J. Phys. A 37 (2004), pp.4501-4526.

[5] P. Buser, Geometry and spectra of compact Riemann surfaces, Progress in Math. 106, Birkhäuser, (1992).

[6] Y. Hashimoto, Arithmetic expressions of Selberg's zeta functions for congruence subgroups, J. Number Theory 122 (2007), pp.324-335.

[7] R. Gangolli, Zeta functions of Selberg's type for compact space forms of symmetric spaces of rank one, Illinois J. Math. 21 (1977), p..1-41.

[8] R. Gangolli and G. Warner, Zeta functions of Selberg's type for some noncompact quotients of symmetric spaces of rank one. Nagoya Math. J. 78 (1980), pp.1-44.

[9] C. F. Gauss, Disquisitiones arithmeticae, Fleischer, Leipzig, (1801). 
[10] S. Geninska and E. Leuzinger, A geometric characterization of arithmetic Fuchsian groups, Duke Math. J. 142 (2008), pp.111-125.

[11] M.C. Gutzwiller, Periodic Orbits and Classical Quantization Conditions, J. Math. Phys. 12 (1971), pp.343-358

[12] G.H. Hardy and E.M. Wright, An introduction to the theory of numbers, Fifth edition, Oxford University Press, 1979.

[13] Y. Hashimoto and M. Wakayama, Splitting density for lifting about discrete groups, Tohoku Math. J. 59 (2007), pp.527-545.

[14] D. Hejhal, The Selberg trace formula of PSL(2, R) I, II, Springer Lec. Notes in Math. 548, 1001 Springer-Verlag $(1976,1983)$.

[15] H. Huber, Zur analytischen Theorie hyperbolischer Raumformen und Bewegungsgruppen I, II, Math. Ann. 138 (1959), 1-26, 142 (1961), pp.385-398 and 143 (1961), pp.463464.

[16] H. Iwaniec, Prime geodesic theorem, J. Reine Angew. Math. 349 (1984), pp.136-159.

[17] V. Lukianov, A mean value theorem for closed geodesics on congruence surfaces, Forum Math. 19 (2007), pp.851-903, http://www.math.tau.ac.il/〜rudnick/students /lukianovthesis.pdf (Ph. D thesis version, Tel-Aviv University, 2005).

[18] W. Luo and P. Sarnak, Number variance for arithmetic hyperbolic surfaces, Commun. Math. Phys. 161 (1994), pp.419-432.

[19] W. Luo and P. Sarnak, Quantum ergodicity of eigenfunctions on $\mathrm{PSL}_{2}(\mathbb{Z}) / H^{2}$, Publ. Math. IHES, 81 (1995), pp.207-237.

[20] W. Luo, Z. Rudnick and P. Sarnak, On Selberg's eigenvalue conjecture, Geom. Funct. Anal. 5 (1995), pp.387-401.

[21] J. Marklof, On multiplicities in length spectra of arithmetic hyperbolic three-orbifolds, Nonlinearity, 9 (1996), pp.517-536.

[22] R. A. Mollin, Quadratics, CRC Press Series on Discrete Mathematics and Its Applications, CRC Press (1995).

[23] M. Peter, The correlation between multiplicities of closed geodesics on the modular surface, Commun. Math. Phys. 225 (2002), pp.171-189.

[24] B. Randol, The length spectrum of a Riemann surface is always of unbounded multiplicity, Proc. Amer. Math. Soc. 78 (1980), pp.455-456.

[25] N. Raulf, Asymptotics of class numbers for progressions and for fundamental discriminants, Forum Math. 21 (2009), pp.221-257.

[26] Z. Rudnick, A central limit theorem for the spectrum of the modular group, Ann. Henri Poincare. 6 (2005), pp.863-883.

[27] P. Sarnak, Class numbers of indefinite binary quadratic forms, J. Number Theory, 15 (1982), pp.229-247.

[28] P. Sarnak, Class numbers of indefinite binary quadratic forms II, J. Number Theory, 21 (1985), pp.333-346.

[29] P. Schmutz, Arithmetic groups and the length spectrum of Riemann surfaces, Duke Math. J., 84 (1996), pp.199-215.

[30] W. Schwarz and J. Spilker, Arithmetical functions, London Mathematica Society LNS 184, Cambridge University Press, 1994. 
[31] A. Selberg, Collected Papers I, Springer-Verlag (1989).

[32] T. Sunada, L-functions in geometry and some applications, Curvature and topology of Riemannian manifolds (Katata, 1985), pp.266-284, Lecture Notes in Math. 1201, Springer, Berlin, 1986.

[33] K. Takeuchi, A characterization of arithmetic Fuchsian groups, J. Math. Soc. Japan 27 (1975), pp.600-612.

[34] A. B. Venkov and P. G. Zograf, Analogues of Artin's factorization formulas in the spectral theory of automorphic functions associated with induced representations of Fuchsian groups, Math. USSR Izvestiya, 21(1983), pp.435-443. 